\title{
Auroral Potential Structures and Current-Voltage Relationship: Summary of Recent Results
}

\author{
P. Janhunen ${ }^{1}$ and A. Olsson ${ }^{2}$ \\ 'Finnish Meteorological Institute, Geophysical Research Division, P. O. Box 503, FIN-00101, Helsinki, Finland \\ ${ }^{2}$ Swedish Institute of Space Physics, Uppsala Division, S-755 91 Uppsala, Sweden
}

Received 6 December 1999; accepted 6 April 2000

\begin{abstract}
Discrete auroral arcs are caused by precipitating accelerated electrons, and there is a lot of observational evidence for the associated potential structure being $U$-shaped below about $15000 \mathrm{~km}$ altitude. However, according to our studies using Polar data at higher altitude, there is a lack of convergent electric field signatures at $\sim 20000-35000 \mathrm{~km}$ and consequently we suggest that the U-shaped potential contours close below this altitude range. To explain this finding, an O-shaped potential model is proposed, together with a maintenance mechanism which involves parallel energisation of middle-energy electrons by waves in the 15000-30000 $\mathrm{km}$ altitude range. Test particle simulations show that the presence of waves brings this "cooperative" model in quantitative agreement with both Polar and low-altitude observations. We also discuss our statistical Freja satellite studies of the observational relationship'between the peak energy (voltage) and the current density in inverted- $V$ regions. The result is that the current and voltage are sometimes correlated in the evening sector events, but almost always anticorrelated in the morning sector. This result, which is very interesting in its own right, may also have some relationship to the potential structure question. $\odot 2001$ Elsevier Science Ltd. All rights reserved
\end{abstract}

\section{Introduction}

It is known that discrete auroral arcs are caused by precipitating magnetospheric electrons, which are accelerated somewhere above the ionosphere in such a way that their energy spectrum has a distinctive peak usually at several keV energy (e.g., Lin and Hoffman, 1979; Bryant, 1999). If one passes through a simple discrete auroral arc in the transverse direction with a sounding rocket or a low-orbiting satellite, the peak energy increases and then decreases, generally reaching a maximum value near the center of the arc. For this reason, the spectra causing discrete arcs are usually said to have an "inverted- $V$ " form.

Correspondence to: $\mathrm{P}$. Janhunen
The inverted- $\mathrm{V}$ electrons are accelerated in the acceleration region at about $6000-13000 \mathrm{~km}$ altitude. There is a lot of evidence that an upward parallel electric field is responsible for the inverted-V electron acceleration (Evans, 1974). It is also known from satellites that pass through the acceleration region that intense perpendicular electric fields exist in this altitude range (Mozer et al. , 1980). Furthermore, the nature of the perpendicular electric field is such that if one forms the line integral of the perpendicular field along the satellite orbit, one obtains a potential value which is generally in agreement with the energy of upward accelerated ion beams (in converging electric field regions) or electron beams (in diverging horizontal electric field regions) observed simultaneously by the particle detectors of the satellite (Marklund, 1993; McFadden et al. , 1998). This has given rise to the theory that the DC electric field is mainly a potential field at this altitude, and the potential contours are U-shaped, the bottom of the "U" corresponding to the upward parallel electric field (Carlquist and Boström, 1970).

Often there are optical undulations or waves moving eastward and westward along the equatorward and poleward edges of an arc (Davis, 1978; Haerendel et al. , 1996). They have been interpreted as reflecting the $\mathbf{E} \times \mathbf{B}$ velocity at and above the acceleration region. The speed of these structures approximately matches the strength of the arc-associated convergent perpendicular fields estimated from the potential drop and the width of the arc, which is another evidence for the applicability of the U-shaped potential picture below about $15000 \mathrm{~km}$ altitude (Haerendel et al. , 1996; Janhunen et al. , 1999b).

This paper, with its limited space, attempts to summarise our recent results concerning auroral potential structures and the current-voltage (CV) relationship. Most of the results are being published in much more detail elsewhere. The paper can therefore be thought of as a "snapshot" of our ongoing work. In this work the methods and instruments vary, but the main aim is to understand the physical processes that are responsible for the creation and maintenance of discrete auroral arcs and inverted-V spectra. The research is motivated by the fact that despite about 30 years of intensive oberva- 


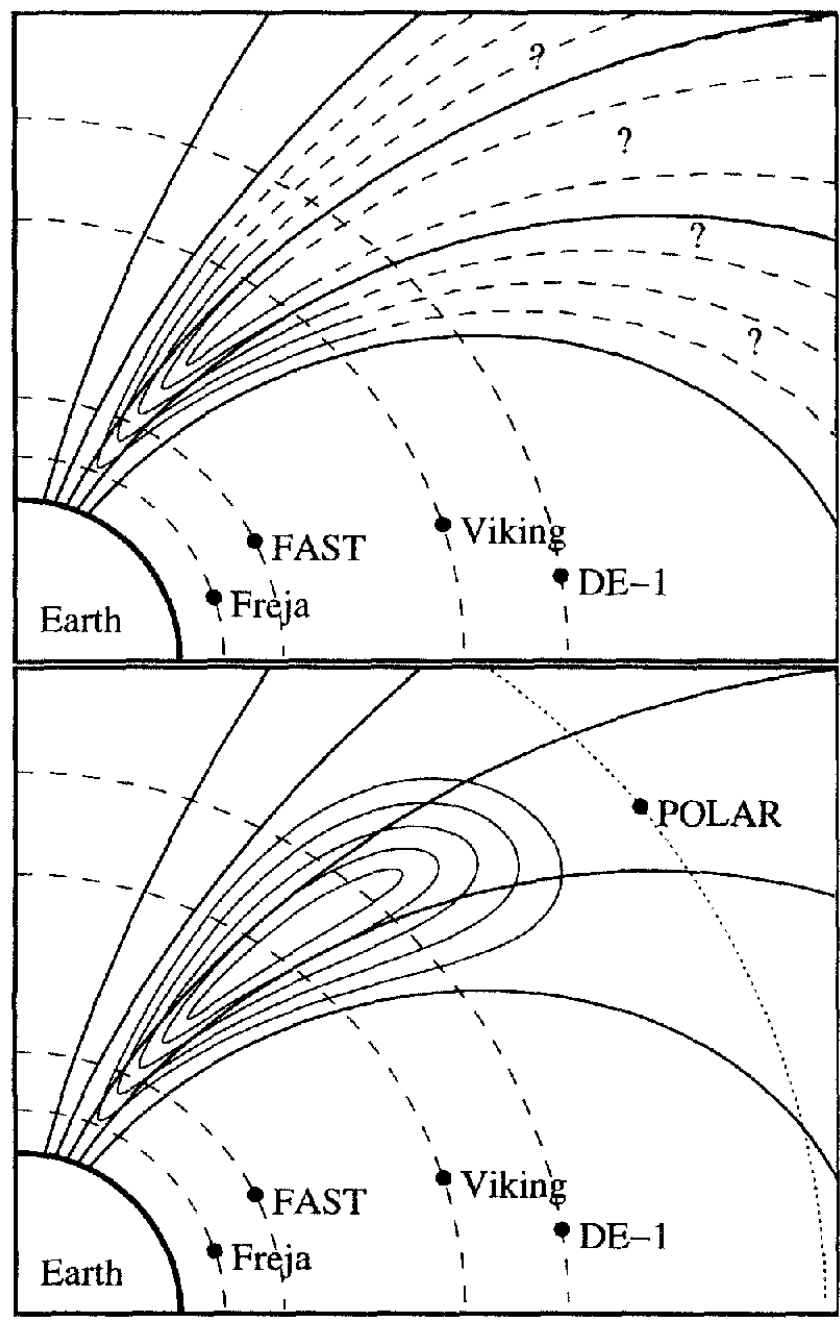

Fig. 1. Top: The knowledge of the acceleration region before POLAR satellite results (Janhunen et al. , 1999a). The potential structure above $15000 \mathrm{~km}$ was poorly known from measurements, but was generally assumed to follow the dashed potential contours. The latitudinal width of the potential structure is exaggerated in order to ease visualisation. Bottom: The postulated O-shaped potential explanation for the observed (Janhunen et al. , 1999a) lack of convergent electric field structures at POLAR altitude.

tional studies, the basic questions about auroral arcs are still open. For example, why arcs are long and narrow, what determines where and when they form, how wide they are and what the peak energy is.

\section{Relative absence of electric field above $25000 \mathrm{~km}$}

There are lots of in situ satellite observations of the auroral region below about $13000 \mathrm{~km}$ altitude, some around 15000 $\mathrm{km}$ (Reiff et al. , 1988), but much fewer higher up, except far in the magnetosphere and close to the equatorial plane. The Polar satellite intersects the northern auroral oval at about $25000-35000 \mathrm{~km}$ altitude, depending on the orbit, and thus can give us valuable information in this poorly known altitude range. Consequently, we studied Polar electric field data using 87 orbits where simultaneous ground-based magnetic observations were also available (Janhunen et al. , 1999a). The idea was to study whether the upright "legs" of the Ushaped potentials can be seen in this altitude range (Figure 1). The result was rather surprising: There was an almost complete lack of evidence for the convergent electric field structures in this altitude range. In a few events, weak (1-2 $\mathrm{kV}$ ) potential wells could be seen, but even they could have been caused by temporal variations. To check that this result was not due to some data processing error, we took a number of Polar southern hemisphere passes (about 6000-10000 $\mathrm{km}$ altitude range, i.e. in the acceleration region) and found many examples of convergent electric fields corresponding to several $\mathrm{kV}$ potential minima, using the same analysis method as for the northern hemisphere passes. To explain the observations, we postulated that the potential contours close below Polar altitude, i.e. that they are $\mathrm{O}$-shaped rather than $\mathrm{U}$ shaped (Figure 1). Finding the potential by integrating along the satellite orbit gives a correct result only in a stationary situation. Therefore substorm-related cases were eliminated in the Polar study, and the conclusions concern only stable arcs. Our conclusions might also apply to dynamically varying arcs, but we cannot verify it from the Polar electric field observations.

Notice that the lower part of an O-shaped potential is Ushaped. Thus the phrase "O-shaped potential" should not be viewed as the opposite of the "U-shaped potential", but rather as a way of completing the U-shaped potential contours. The O-shaped potential model is, however, markedly different from a model in which the field lines are equipotentials above the acceleration region and the U-shaped contours continue all the way to the equatorial plane, closing only in the opposite hemisphere in a (presumably symmetric) U-shaped structure. Let us call the theory where the potential in the acceleration region is U-shaped but where the closing of the contours is left open the weak U-shaped model and the theory where the U-shaped contours close only in the opposite hemisphere the strong $U$-shaped model. The strong U-shaped model implies the weak one, but not vice versa. As mentioned above, satellite observations below about 15000 $\mathrm{km}$ support the weak $\mathrm{U}$-shaped model. The $\mathrm{O}$-shaped model is compatible with the weak U-shaped model. On the contrary, to our knowledge there are no observational data directly supporting the strong U-shaped model, and the strong U-shaped model is in disagreement with the Polar results and the O-shaped model.

\section{The "cooperative" model}

In an O-shaped potential barrier, electrons are not accelerated but the low energy part of the magnetospheric electron population is filtered out (reflected back) before hitting the ionosphere (Janhunen et al. , 1999a). It turns out that in case of a Maxwellian source plasma, the filtering due to a potential barrier produces the same functional form for the low altitude spectrum as an acceleration by a potential drop. Thus, there is no problem in explaining the low-altitude inverted- 
$\mathrm{V}$ electron spectra by the O-shaped model, provided that the electron energy flux is large enough. However, our current understanding is that in most cases, the magnetospheric thermal electron flux is insufficient to explain the observed intensity at low altitude. This conclusion is mainly based on our conjugate studies between Polar and FAST which are being published elsewhere.

To resolve the intensity problem we propose that above the acceleration region, there are plasma waves causing parallel energization of medium-energy electrons (roughly 50$400 \mathrm{eV}$ ). If the parallel energy of an electron is increased by a wave-particle interaction, its pitch angle is decreased and its mirror point moves closer to the ionosphere. The net effect is a buildup of a negative charge cloud of magnetospheric electrons at low altitudes (Alfvén and Fälthammar, 1963). Close to the ionosphere, the cloud is easily neutralized by a displacement of cold ionospheric electrons away from the cloud or by an adjustment in the cold ion density. At some altitude, however, the density of the ionospheric plasma is not sufficient for a complete neutralization, thus the cloud will have, after a partial neutralization, a maximum charge density at a certain altitude. Because of Poisson's equation, the potential corresponding to such a negative charge cloud looks O-shaped.

The center of the O-shaped potential is likely to form at an altitude which is slightly below the region where most of the electron energisation by waves is taking place. Thus, the DC electric field points downward in the region where the waves are giving parallel boosts to electrons, and thus the waves must work against the electric field when giving electrons a downward boost. To make further conclusions one has to assume something about the nature of the waves; let us assume that the wave-particle interaction is resonant, i.e. that only those electrons whose parallel velocity is close to the phase velocity of the waves are affected, and that this velocity corresponds to the middle-energy range $(50-400 \mathrm{eV}$, roughly) when transformed to electron energy. The net effect of the downward DC field is then to keep the electrons in the resonant velocity range for a longer time than would otherwise occur, thus helping to extract a larger amount of energy from the waves to the electrons than what would happen without the DC field. That a downward electric field could increase the net amount of particle energisation may sound paradoxical, but this is what the test particle simulation shows. What happens is that the energy extracted from the waves goes mostly to increase the electron potential energy (the waves "push the electrons up a potential hill"). When electrons reach the top of the hill, they are accelerated downward (potential energy is turned to kinetic energy) exactly as in any weak $\mathrm{U}$-shaped model (for the definition of weak and strong U-shaped models, see the end of Section 2).

Thus, we propose that an $\mathrm{O}$-shaped potential is formed as a consequence of wave-particle interactions (Figure 2) and that the potential helps increase the effectiveness of the waves and is also responsible for the functional form of the low-altitude inverted-V electron spectrum. According to our test particle simulations (Janhunen and Olsson, 2000), the "cooperative"

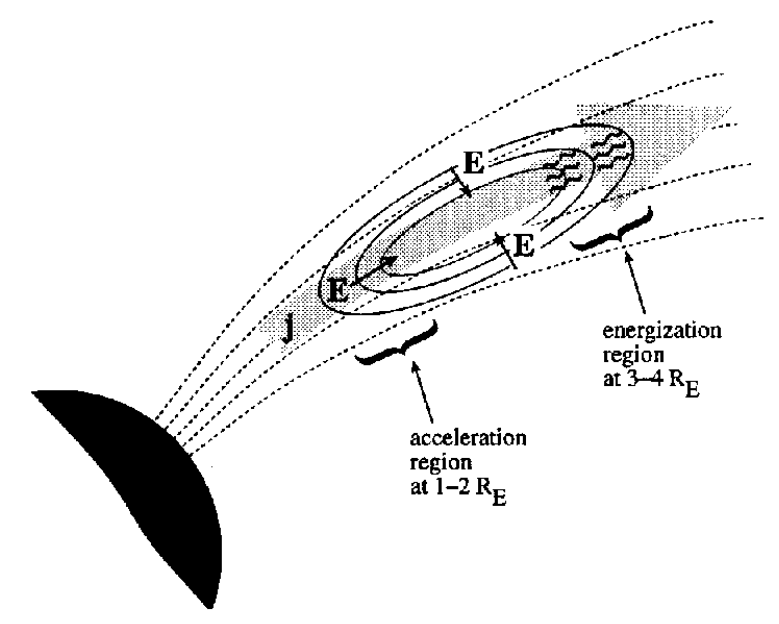

Fig. 2. The "cooperative" model for inverted-V auroral acceleration and self-consistent maintenance of an $\mathrm{O}$-shaped potential structure.

model (named so because waves and the potential structure cooperate, both being essential) can explain the observed low altitude spectra quantitatively, provided that suitable waves exist in the $\sim 15000-30000 \mathrm{~km}$ altitude range. One interesting feature of the model is that there are separete energisation and acceleration regions for the electrons (Figure 2).

To be relevant, the "cooperative" model requires the presence of plasma waves giving parallel boosts to middle-energy electrons in the $\sim 15000-30000 \mathrm{~km}$ altitude range. The presence and precise nature of such waves is still an open question, but we have seen promising events in Polar PWI data: intense broadband electrostatic wave bursts correlated with simultaneous middle-energy electron $T_{\|}>T_{\perp}$ anisotropies. These findings will be published in detail elsewhere.

\section{Statistical current-voltage relationship}

The relationship between the peak energy and the field-aligned current (FAC) density in inverted-V events is usually called the current-voltage (CV) relationship (Olsson et al. , 1996,

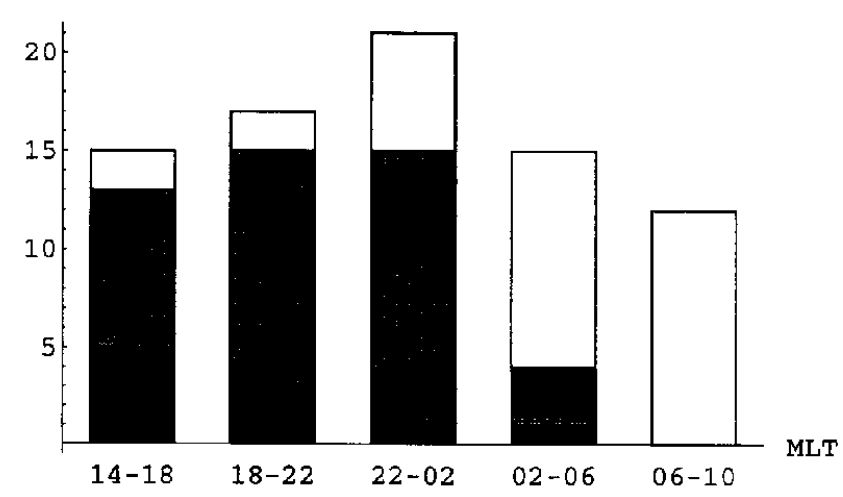

Fig. 3. The number of Freja U-events (black), C-events (gray) and O-events (white) in each MLT sector. In U-events the current and voltage are correlated, in C-events they are not, and in O-events they are anticorrelated. 
1998). Here we consider the question from a purely observational viewpoint: how the current $j$ and the voltage ( $\sim$ peak energy) $V$ are correlated. We study 81 inverted- $V$ events using the Freja satellite $\mathrm{F} 7$ instrument (Boehm et al. , 1994) for estimating both current and voltage, using $31 \mathrm{~ms}$ time resolution. The events are distributed in all MLT sectors, except in the noon sector where no inverted-V events are found (Lin and Hoffman, 1979). The results are reported in detail elsewhere (Olsson and Janhunen, 2000a,b), but a main finding is that when all events are considered together, the current $j$ does not, on the average, depend on the voltage $V$ (Olsson and Janhunen, 2000a). Even more interesting is that there is clear MLT dependence (Olsson and Janhunen, $2000 \mathrm{~b}$ ). Let us label events where the current and voltage are positively correlated by " $U$ ", events where they are not correlated by " $\mathrm{C}$ ", and events where they are anticorrelated by " $O$ ". In Figure 3 we show the number of each class of events for each MLT sector. We see that in the evening sector, Utype events are rather common, but even there the majority of events belongs to the $C$-class. Morning sector inverted-V events seem to consist almost exclusively of $\mathrm{O}$-class events. This is an interesting result in its own right. Previous studies made with much smaller datasets have generally suggested linearly correlated current and voltage (Lyons et al. , 1979).

It is still an open question what is the relationship between the auroral potential structure and the statistical CVrelationship results. One possibility is that anticorrelative and constant $\mathrm{CV}$-relationships ( $\mathrm{O}$ and $\mathrm{C}$ events) correspond to stable arcs and predominantly $\mathrm{O}$-shaped potential structures, while the rarer evening sector correlative CV-relationship events (U-events) correspond to either strong U-shaped potential models (for definition of the term see Section 2) or dynamical cases where inductive electric fields are so important that speaking of a potential structure no longer makes sense. It fits in this picture that auroral surge features related to substorms and other auroral activations typically contain higher peak energies than stable arcs; they are also more dynamical and occur mostly in the premidnight sector.

\section{Summary}

Our recent observations indicate that potential contours that look U-shaped in the acceleration region do not continue up to the equatorial plane, but close at a much lower altitude, below $25000 \mathrm{~km}$ or so. (The altitude figures given in this paper should be taken as a rough estimate only.) This implies that there is a downward DC electric field above the acceleration region. To explain this finding without contradicting the existing low-altitude observational data we suggest the "cooperative" model for auroral acceleration. In this model, electrons are energised by random parallel boosts of resonantly interacting plasma waves above $15000 \mathrm{~km}$. An O-shaped potential is responsible for temporarily storing the transferred energy in potential energy form and transforming it into electron kinetic energy in the acceleration region, where the process is exactly as in the classical weak $\mathrm{U}$-shaped model. This model can explain both low and high altitude observations quantitatively, provided that suitable waves exist in the $\sim 15000$ $30000 \mathrm{~km}$ altitude range. Our results of the $\mathrm{CV}$-relationship indicate that the morningside inverted- $\mathrm{V}$ events are different from the eveningside ones, because in the morningside, the current and the voltage are anticorrelated. This result may well have something to do with the potential structure question. In the future, observational studies should look for evidence on suitable plasma waves in the $\sim 15000-30000$ $\mathrm{km}$ range. Likewise, the free energy source for the waves, and consequently for the whole auroral acceleration process, should be identified in the magnetosphere.

Acknowledgements. We thank W.K. Peterson, J.S. Pickett, J.P. McFadden, C.W. Carlson, C.T. Russell, F.S. Mozer, C.A. Kletzing, J.H. Clemmons and T.J. Hughes for providing data and interpretation hints for our studies, and H. Laakso, K. Kauristie, A.I. Eriksson and many other collagues for useful discussions and practical help. Annika Olsson's work is supported by the Swedish Natural Science Research Council and the Knut and Alice Wallenberg Foundation.

\section{References}

Alfvén, H. and C.G. Fälthammar, Cosmical Electrodynamics, Clarendon, Oxford, 1963.

Boehm, M., G. Paschmann, J. Clemmons, H. Höfner, R. Frenzel , M. Ertl, G. Haerendel, P. Hill, H. Lauche, L. Eliasson, and R. Lundin, The TESP electron spectrometer and correlator (F7) on Freja Space. Sci. Rew, 70, 509-540, 1994.

Bryant, D.A., Electron acceleration in the aurora and beyond, Institute of Physics Publishing, Bristol, 1999.

Carlqvist, P. and R. Boström, Space-charge regions above the aurora, J. Geophys. Res., 75, 7140-7146, 1970.

Davis, T.N., Observed characteristics of auroral forms, Space Sci. Rev., 22, 77-113, 1978.

Evans, D.S., Precipitating electron fluxes formed by a magnetic field aligned-potential difference, J. Geophys. Res., 79, 2853-2858, 1974.

Haerendel, G., B.U. Olipitz, S. Buchert, O.H. Bauer, E. Rieger and C. La Hoz, Optical and radar observations of auroral arcs with emphasis on small-scale structures, J. Atmos. Terr. Phys., 58, 71-83, 1996.

Janhunen, P., A. Olsson, F.S. Mozer and H. Laakso, How does the U-shaped potential close above the acceleration region? A study using Polar data, Ann. Geophysicae, 17, 1276-1283, 1999.

Janhunen, P., and A. Olsson, New model for auroral acceleration: $O$-shaped potential structure cooperating with waves, Ann. Geophysicue, in press, 2000.

Janhunen, P., A. Olsson, O. Amm and K. Kauristie, Characteristics of a stable arc based on FAST and MIRACLE observations, Ann. Geophysicae, $18,152-160,2000$

Lin, C.S, and R.A. Hoffman, Characteristics of the inverted-V event, J. Geophys. Res., 84, 1514-1525, 1979.

Lyons, L.R., D.S. Evans and R. Lundin, An observed relation between magnetic field aligned electric fields and downward electron energy fiuxes in the vicinity of auroral forms, J. Geophys. Res., 84, 457-461, 1979.

Marklund, G., Viking investigations of auroral electrodynamic processes, $J$. Geophys. Res., 98, 1691-1704, 1993.

McFadden, J.P., C.W. Carlson, R.E. Ergun, F.S. Mozer, M. Temerin, W. Peria, D.M. Klumpar, E.G. Shelley, W.K. Peterson, E. Moebius, L. Kistler, R. Elphic, R. Strangeway, C. Cattell and R. Pfaff, Spatial structure and gradients of ion beams observed by FAST, Geophys. Res. Lett., 25, 20212024, 1998.

Mozer, F.S., C.A. Cattell, M.K. Hudson, R.L. Lysak, M. Temerin, and R.B. Torbert, Satellite measurements and theories of low altitude auroral particle acceleration, Space Sci. Rev., 27, 155-213, 1980. 
Olsson, A., A.I. Eriksson and P. Janhunen, On the current-voltage relationship in auroral breakups and westwards-travelling surges, Ann. Geophysicue, 14, 1265-1273, 1996.

Olsson, A., Andersson, L., Eriksson, A.I., Clemmons, J., Erlandsson, R.E., Reeves, G., Hughes, T. and Murphree, J.S., Freja studies of the currentvoltage relation in substorm related events, J. Geophys. Res., 103, 42854301, 1998.

Olsson, A., and P. Janhunen, A statistical study of nightside inverted-V events using Freja electron data: Implications for the current-voltage re- lationship, J. Atmos. Solar Terr. Phys., 62,, 81-92, 2000.

Olsson, A., and P. Janhunen, Difference in the current-voltage relationships between dawn and duskside inverted-V events, J. Geophys. Res., 105, $5373-5380,2000$.

Reiff, P.H., H.L. Collin, J.D. Craven, J.L. Burch, J.D. Winningham, E.G. Shelley, L.A. Frank and M.A. Friedman, Determination of auroral electrostatic potentials using high-and low-altitude particle distributions, $J$. Geophys. Res., 93, 7441-7465, 1988. 